\title{
Water reuse and growth inhibition mechanisms for cultivation of microalga Euglena gracilis
}

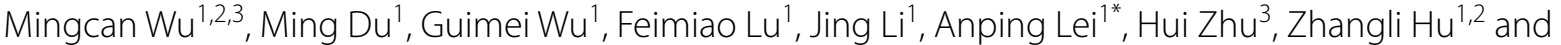 \\ Jiangxin Wang ${ }^{1 *}$ (D)
}

\begin{abstract}
Background: Microalgae can contribute to more than $40 \%$ of global primary biomass production and are suitable candidates for various biotechnology applications such as food, feed products, drugs, fuels, and wastewater treatment. However, the primary limitation for large-scale algae production is the fact that algae requires large amounts of fresh water for cultivation. To address this issue, scientists around the world are working on ways to reuse the water to grow microalgae so that it can be grown in successive cycles without the need for fresh water.
\end{abstract}

Results: In this study, we present the results when we cultivate microalgae with cultivation water that is purified and reused. Specifically, we purify the cultivation water using an ultrafiltration membrane (UFM) treatment and investigate how this treatment affects: the biomass and biochemical components of the microalgae; characteristics of microalgae growth inhibitors; the mechanism whereby potential growth inhibitors are secreted (followed using metabolomics analysis); the effect of activated carbon (AC) treatment and advanced oxidation processes (AOPs) on the removal of growth inhibitors of Euglena gracilis. Firstly, the results show that E. gracilis can be only cultivated through two growth cycles with water that has been filtered and reused, and the growth of E. gracilis is significantly inhibited when the water is used a third time. Secondly, as the number of reused water cycles increases, the $\mathrm{Cl}^{-}$concentration gradually increases in the cultivation water. When the $\mathrm{Cl}^{-}$concentration accumulates to a level of fivefold higher than that of the control, growth of $E$. gracilis is inhibited as the osmolality tolerance range is exceeded. Interestingly, the osmolality of the reused water can be reduced by replacing $\mathrm{NH}_{4} \mathrm{Cl}$ with urea as the source of nitrogen in the cultivation water. Thirdly, E. gracilis secretes humic acid (HA) - which is produced by the metabolic pathways for valine, leucine, and isoleucine biosynthesis and by linoleic acid metabolism -into the cultivation water. Because HA contains large fluorescent functional groups, specifically extended $\pi$ (pi)-systems containing $C=C$ and $C=O$ groups and aromatic rings, we were able to observe a positive correlation between $\mathrm{HA}$ concentration and the rate of inhibition of E. gracilis growth using fluorescence spectroscopy. Moreover, photosynthetic efficiency is adversely interfered by HA, thereby reductions in the synthetic efficiency of paramylon and lipid in E. gracilis. In this way, we are able to confirm that HA is the main growth inhibitor of E. gracilis. Finally, we verify that all the HA is removed or converted into nutrients efficiently

\footnotetext{
*Correspondence: bioaplei@szu.edu.cn; jxwang@szu.edu.cn

${ }^{1}$ Shenzhen Key Laboratory of Marine Bioresource and Eco-Environmental

Science, Shenzhen Engineering Laboratory for Marine Algal

Biotechnology, Guangdong Provincial Key Laboratory for Plant

Epigenetics, College of Life Sciences and Oceanography, Shenzhen

University, Shenzhen 518060, China

Full list of author information is available at the end of the article
}

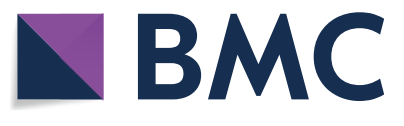

(c) The Author(s) 2021. This article is licensed under a Creative Commons Attribution 4.0 International License, which permits use, sharing, adaptation, distribution and reproduction in any medium or format, as long as you give appropriate credit to the original author(s) and the source, provide a link to the Creative Commons licence, and indicate if changes were made. The images or other third party material in this article are included in the article's Creative Commons licence, unless indicated otherwise in a credit line to the material. If material is not included in the article's Creative Commons licence and your intended use is not permitted by statutory regulation or exceeds the permitted use, you will need to obtain permission directly from the copyright holder. To view a copy of this licence, visit http://creativeco mmons.org/licenses/by/4.0/. The Creative Commons Public Domain Dedication waiver (http://creativecommons.org/publicdomain/ zero/1.0/) applies to the data made available in this article, unless otherwise stated in a credit line to the data. 
by AC or UV/ $\mathrm{H}_{2} \mathrm{O}_{2} / \mathrm{O}_{3}$ treatments, respectively. As a result of these treatments, growth of $E$. gracilis is restored (AC treatment) and the amount of biomass is promoted $\left(\mathrm{UV} / \mathrm{H}_{2} \mathrm{O}_{2} / \mathrm{O}_{3}\right.$ treatment).

Conclusions: These studies have important practical and theoretical significance for the cyclic cultivation of E. gracilis and for saving water resources. Our work may also provide a useful reference for other microalgae cultivation.

Keywords: Microalgae, Euglena gracilis, Water reuse, Growth inhibitor, Humic acid, Metabolomics

\section{Background}

Unicellular eukaryotic microalgae are a diverse and ubiquitous group of plants that are a promising biomass source and a biological feedstock $[1,2]$. However, the use of microalgae as a feedstock is limited because a large amount of water is needed for its cultivation, and this negatively affects economic viability and environmental sustainability [3]. Wastewater treatment is currently being used to recycle the water that is necessary for microalgae cultivation [4]. It has been reported that to produce $1 \mathrm{~kg}$ of algae biomass, $1564 \mathrm{~L}$ of water are required under pond conditions [5]. Reusing cultivation water can reduce water usage and nutrient requirements as well as the need for algal wastewater treatment [3]. Consequently, water reuse after algae harvesting is essential for the economic viability of the microalgae industry and for environmental sustainability.

The effects of water reuse on algae growth are different across algae taxa [6]. The most researched taxa are green algae, diatoms, cyanobacteria, haptophytes, eustigmatophytes, chrysophytes, and xanthophytes [3]. However, among the genus of Euglena spp. algae, especially, there have been no reports of E. gracilis cultivation with reused water. E. gracilis is a unicellular flagellated alga characterized by the absence of a cell wall. It produces a wide variety of bioactive compounds such as paramylon, carotenoids, tocopherols, euglenophycin, and lipids. It has tremendous potential for metabolic engineering and commercialization [7]. Therefore, a deeper understanding of water reuse in the cultivation of the microalga $E$. gracilis, and the mechanisms underlying its growthinhibiting secretions, are urgently needed.

After the microalgae assimilate nutrients ions, unabsorbed counter ions such as $\mathrm{Cl}^{-}, \mathrm{Na}^{+}$, and $\mathrm{K}^{+}$from $\mathrm{NH}_{4} \mathrm{Cl}, \mathrm{NaHCO}_{3}$, and $\mathrm{KH}_{2} \mathrm{PO}_{4}$, respectively, can accumulate in the cultivation water. When these ions accumulate, the osmotic pressure of the cultivation water increases, thereby inhibiting microalgae growth $[8,9]$. Therefore, finding a suitable medium, which can balance the osmotic pressure between microalgae and cultivation water, is important for improving the effects of water reuse.

In addition to accumulated ions, excreted metabolites, such as dissolved organic matter (DOM) from microalgae is considered to be the main cause of negative biomass growth [3, 6, 10]. For Scenedesmus sp. LX1, DOM concentrations between 6.4 and $25.8 \mathrm{mg} / \mathrm{L}$ in reused water resulted in a decrease in the maximum algae cells density and the maximum growth rate by $50-80 \%$ and $35-70 \%$, respectively [11]. The DOM in that study was classified into two fractions: hydrophobic or hydrophilic. Each of these fractions was further classified as acids, neutrals, or bases for a total of six fractions. In that study, all six fractions showed inhibited algal growth. Moreover, Lu et al. [10] who also used this fractionation approach, reported that the DOM of Scenedesmus acuminatus in the reused water included palmitic acid and octadecanoic acid, both of which inhibited the growth of this algae species. Although many studies have attempted to characterize the growth inhibitor present in DOM, it is unclear which major metabolic pathways within microalgae cells regulate and secrete these inhibitory substances into the cultivation water.

Recently, many researchers have tried to use traditional methods of wastewater treatment on microalgae cultivation water. Zhang et al. [12] reported that the removal of DOM with activated carbon (AC) in the reused water of cultivated Nannochloropsis oceanica moderately reduced growth inhibition and lipid accumulation. Moreover, the AC treatment significantly increased the final dry weight of $S$. acuminatus to $2.33 \pm 0.04 \mathrm{~g} / \mathrm{L}$, which was almost the same as the dry weight obtained after growth in fresh media [13]. Advanced oxidation processes (AOPs) are another type of treatment technology in which organic pollutants are destroyed by powerful oxidizing agents $[14,15] . \mathrm{O}_{3}, \mathrm{UV} / \mathrm{H}_{2} \mathrm{O}_{2}$ have been successfully applied in the treatment reused water for the cultivation of Scenedesmus sp. LX1 [16] and S. acuminatus GT-2 [17], respectively. To date, few studies have investigated the removal of DOM by AC or AOPs in reused water of cultivated $E$. gracilis.

In this study, the main objectives were: (1) to identify the effect of treatment with ultrafiltration membrane (UFM) on the biomass and biochemical components of cultivated E. gracilis; (2) to identify the characteristics of the growth inhibitors in reused water and uncover the mechanism whereby potential inhibitors are secreted by E. gracilis via metabolomics analysis; (3) and to evaluate the effect of AC and AOPs on the removal of growth inhibitors. 


\section{Results and discussion}

\section{Effects of reused water on the growth of $E$. gracilis}

Since the UFM can cut off substances with a molecular weight $\geq 50 \mathrm{kDa}$, viruses, bacteria, macromolecular proteins, polysaccharides, and other substances can be filtered out $[10,12]$, so only unconsumed ions and DOM remain in the reused water. This study found that the DW of algae cells gradually decreased in the reused water with successive cycles. The DW of algae with each cycle of cultivation decreased by $13.1 \%$ (UFM-R1, $p<0.05$ ), 28.6\% (UFM-R2, $p<0.05$ ), and 79.2\% (UFM$\mathrm{R} 3, p<0.01)$ compared to the control group on the last day of cultivation (Fig. 1). By the third cycle of cultivation, the growth of algae cells had been severely inhibited. This suggests that the increase in the presence of growth inhibitors with successive cycles of water reuse reduces algal growth beyond a tolerable range. This phenomenon is similar to the growth inhibition observed for other microalgae such as $S$. acuminatus [10], Chlorella. SDEC-18 [18], and N. oceanica [12]. We postulate that accumulated ions and algae cells secretions of DOM in the reused water are the main factors that inhibit the growth of E. gracilis.
The effect of accumulated ions on the growth of E. gracilis Microalgae can selectively absorb some types of ions from inorganic nutrients and assimilate them into their own organic matter. But some ions such as $\mathrm{Cl}^{-}$and $\mathrm{Na}^{+}$, cannot be absorbed by microalgae. These residual ions, which accumulate in reused water, can destroy the balance of osmolality of the microalgae, thereby inhibiting their growth $[8,9]$. This study found that when algae cells were cultivated in more than eightfold the concentration of PEM medium $\left(\mathrm{NH}_{4} \mathrm{Cl}\right.$ as the nitrogen source) compared to the control, the relative cell density was lower, algal cytochromes were almost absent, and the algae cells were elongated (Fig. 2a, b). When the concentration of the culture medium was within fivefold of the control medium, the DW of algal cells was about $2.7 \mathrm{~g} / \mathrm{L}$, which was not significantly different from the control group $(p>0.05)$. However, when the concentrations of the medium were increased to above fivefold that of the control medium, the DW gradually decreased. In fact, when the concentration of the medium was increased by eight-, nine-, and tenfold, the DW of cells decreased by $81.3 \%(p<0.01), 85.0 \%(p<0.01)$, and $92.5 \%(p<0.01)$, respectively (Fig. 2c). In addition, the $\mathrm{Cl}^{-}$concentration was increased as the medium concentration increases,

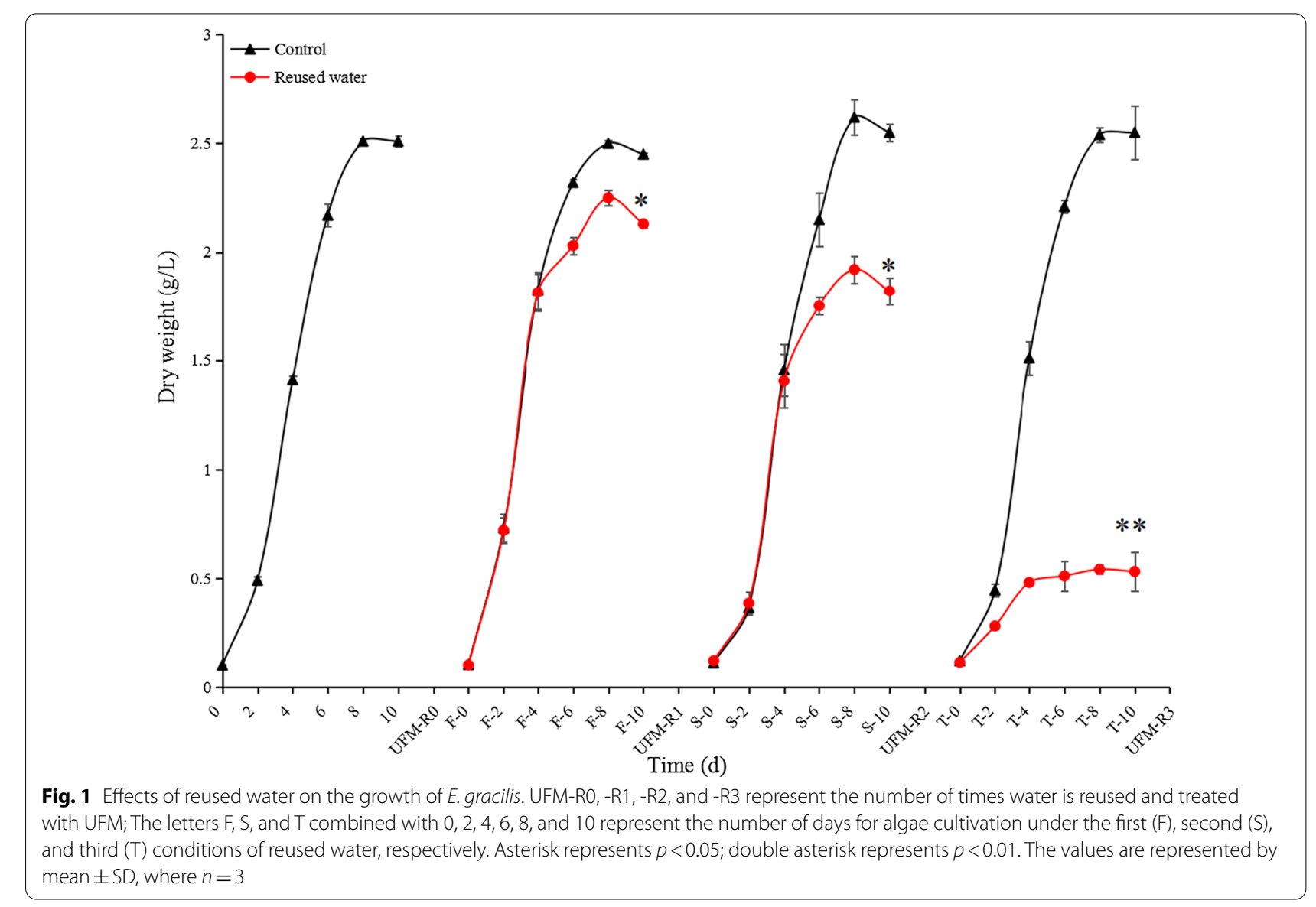




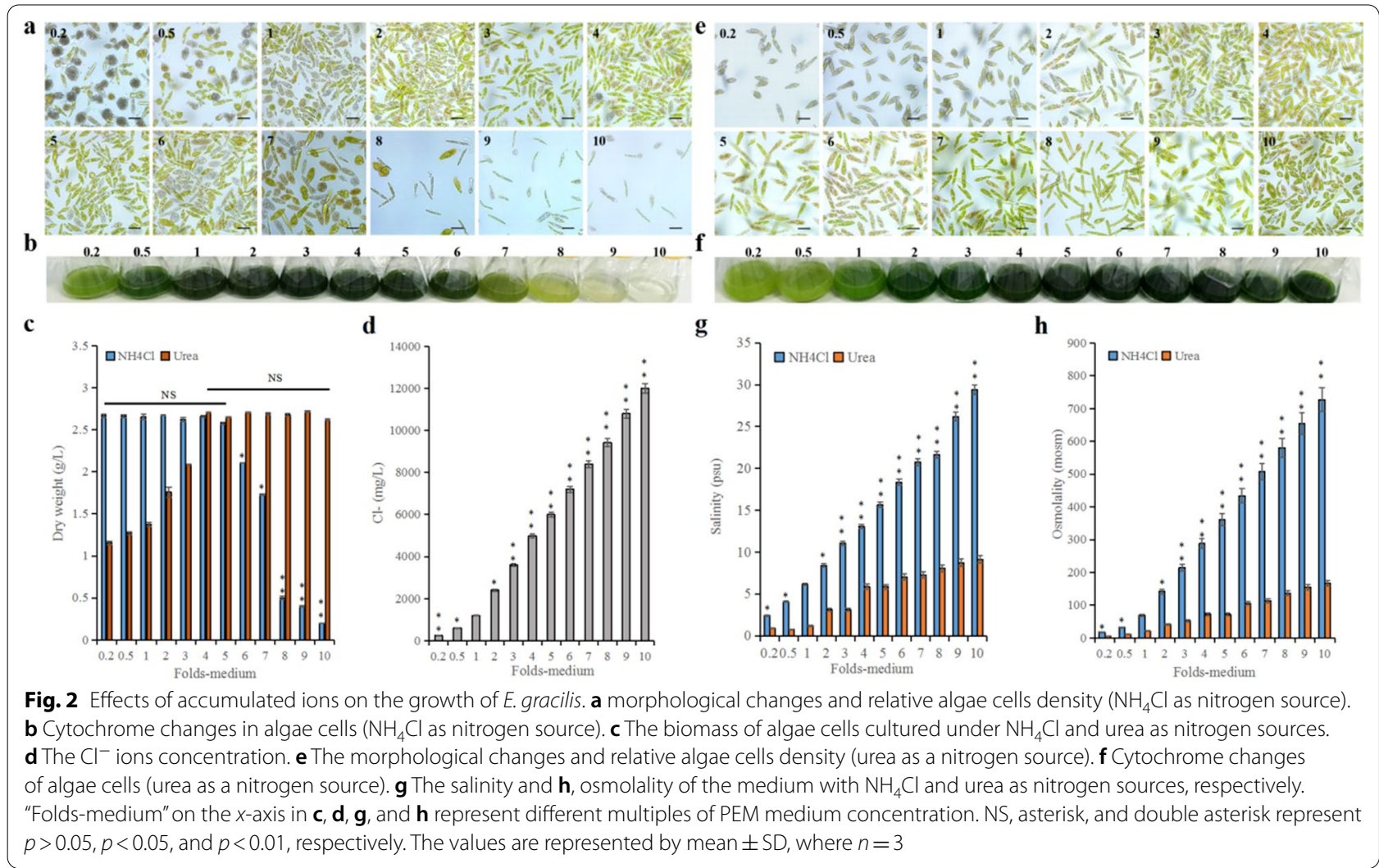

the maximal concentration was reached $11,996.4 \mathrm{mg} / \mathrm{L}$ in the tenfold medium (Fig. 2d), suggesting that accumulated $\mathrm{Cl}^{-}$in the medium may be a key growth inhibitor of E. gracilis.

To prove the above hypothesis, we used urea instead of $\mathrm{NH}_{4} \mathrm{Cl}$ as the nitrogen source (equal nitrogen content) and found that as the concentration of urea increased, the DW of algae cells increased significantly. When the concentration of the culture medium was four to tenfold, the biomass of algal cells was stably maintained at about $2.7 \mathrm{~g} / \mathrm{L}$, and there was no significant difference among them $(p>0.05)$ (Fig. 2c). In addition, when urea was used as a nitrogen source, the cells appeared to be fuller. With an increase in the concentration of the culture medium, the relative cells density increased and the relative content of chlorophyll gradually increased as well (Fig. 2e, f). At the same time, the salinity and osmolality in the medium were much lower than those of the medium using $\mathrm{NH}_{4} \mathrm{Cl}$ as the nitrogen source. For example, the salinities at tenfold medium concentration were $29.4 \mathrm{psu}$ versus $9.1 \mathrm{psu}$ for $\mathrm{NH}_{4} \mathrm{Cl}$ versus urea, respectively. The osmolalities under these conditions were 727.0 mosm versus 167.0 mosm for $\mathrm{NH}_{4} \mathrm{Cl}$ versus urea, respectively. The salinity value for $\mathrm{NH}_{4} \mathrm{Cl}$ was 3.2-fold greater than urea (Fig. $2 \mathrm{~g}, p<0.01$ ), and the osmolality for $\mathrm{NH}_{4} \mathrm{Cl}$ was 4.3-fold greater than urea (Fig. $2 \mathrm{~h}, p<0.01$ ). These results show that the growth of algae cells was not inhibited with a fivefold increase in salinity $(<15.6 \mathrm{psu})$ and osmolality $(<361.1$ mosm $)$ of the medium, and we can confirm that during the UFM-R3 culture cycle, the growth of $E$. gracilis was not hindered by the accumulated ions in the reused water. This phenomenon has also been confirmed by the cultivation of $S$. acuminatus in reused water [10, 19]. Our work also showed that the growth of E. gracilis had a certain tolerance range to ions. If this tolerance range was exceeded, the growth of E. gracilis was inhibited. In addition, it was determined that the traditional medium PEM with $\mathrm{NH}_{4} \mathrm{Cl}$ as the nitrogen source was not suitable for the continuous recycling of cultivation water or for batch-fed cultivation of E. gracilis (such as heterotrophic batch-fed fermentation). When urea is used, it serves as an ideal nitrogen source because it reduces the salinity and osmolality in the culture medium.

\section{Identification of growth inhibitors in E. gracilis secretions}

The growth of microalgae is not affected by certain osmotic pressures for reused water, so growth inhibitors may exist in the DOM secreted by microalgae. However, some DOM can promote the growth of microalgae while some have an inhibitory effect on microalgae growth [3], so further study of these DOM characteristics is required. This study also found that E. gracilis 
continuously secreted DOM during the culture process. By the time UFM-R3 was reached, the DOM concentration was $189.21 \mathrm{mg} / \mathrm{L}$, while the control group contained only $54.92 \mathrm{mg} / \mathrm{L}$ DOM, a 3.4 -fold difference $(p<0.01)$ (Fig. 3a). This indicates that, at elevated concentrations, DOM may have an inhibitory effect on the growth of $E$. gracilis.

3D-FEEM fluorescence spectroscopy is fast and has excellent selectivity and sensitivity for fluorescent substances [20]. Therefore, in this study, this technique was used to identify the types of DOM secreted by algae cells. Chen et al. [21] used 3D-FEEM fluorescence spectroscopy to identify the following substances in the DOM present in cultivation water: aromatic proteins (AP), fulvic acid-like substances (FA), soluble microbial by product-like material (SMBM), and HA. We used those assignments to determine which types of DOM were present in our cultivation water samples (they are labeled with roman numerals in the spectrum in Fig. 3b. See caption b). It can be seen from Fig. 3b that the abundance of organic compounds with fluorescent signals in the reused water from high to low as: HA, SMBM, FA, and AP. Our spectra showed that HA was the potential main type of DOM present in E. gracilis secretions.

In order to further identify the growth inhibitors, we divided the DOM into six major fractions using fractional distillation (Fig. 3c). The percentages from high to low were: $\mathrm{HiN}(32 \%), \mathrm{HoA}(27 \%), \mathrm{HoN}(25 \%), \mathrm{HiB}(7 \%)$, $\mathrm{HoB}(6 \%)$, and $\mathrm{HiA}(3 \%)$. From this result, we know that the DOM is mainly composed of $\mathrm{HiN}, \mathrm{HoA}$, and $\mathrm{HoN}$, suggesting that HA, a potential inhibitor of E. gracilis, is composed of these organic acids. We also know that the slope of peaks in a UV spectrum (at $254 \mathrm{~nm}$, given in AU/ $\mathrm{cm}$ ) for organic matter represents the content of organic functional groups that contribute to fluorescence, such as $\mathrm{C}=\mathrm{C}$ bonds, $\mathrm{C}=\mathrm{O}$ bonds, and aromatic rings. The importance of ultraviolet spectra for detecting pollutants in the water treatment process was described by Altmann
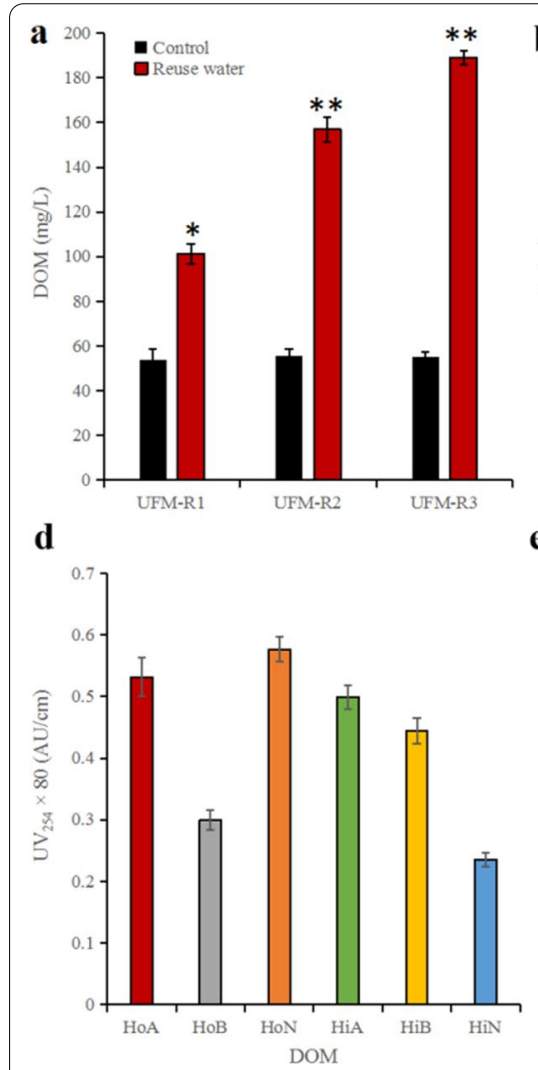

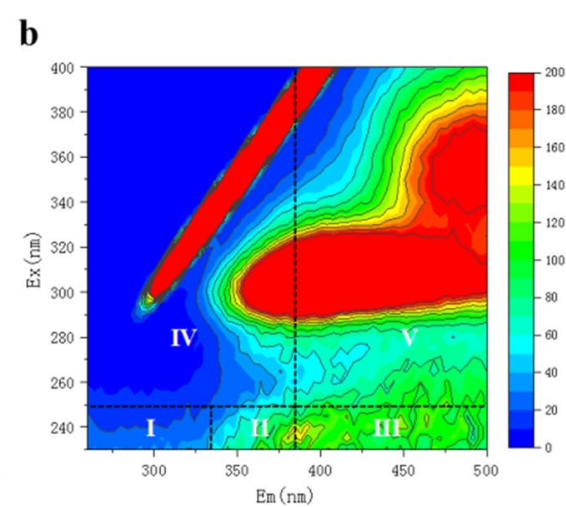

e

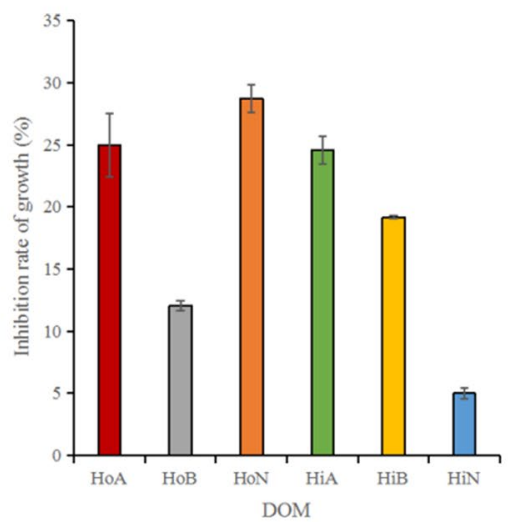

c

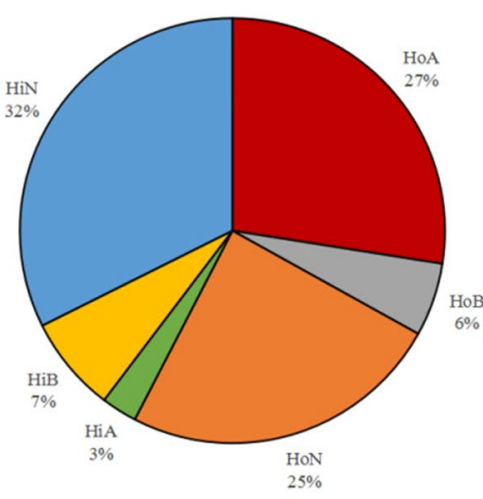

f

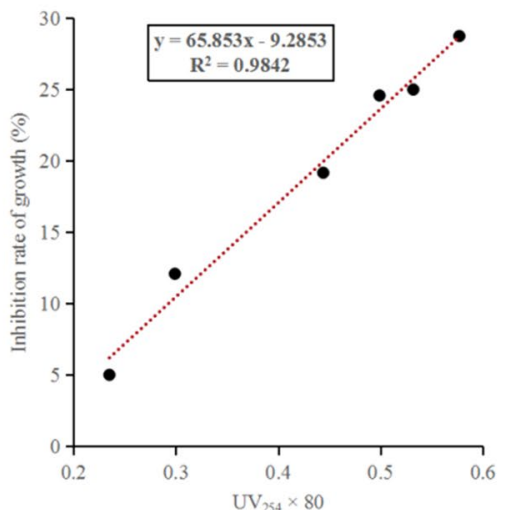

Fig. 3 Identification of growth inhibitors in E. gracilis secretions. a The content of DOM in reused water by using ultrafiltration membrane treatment. b Three-dimensional (3D) fluorescence excitation-emission matrix (FEEM) spectra of DOM (I and II, AP, aromatic proteins; III, FA, fulvic acid-like, IV, SMBM, soluble microbial by product-like material; $V, H A$, humic acid). c Percent of 6 DOM fractions from reused water of E. gracilis. $\mathbf{d}$ UV 254 of 6 DOM fractions in 80-fold medium (HoB hydrophobic bases, HiN hydrophilic neutrals, HoA hydrophobic acids, HiB hydrophilic bases, HiA hydrophilic acids, HoN hydrophobic neutrals). e The inhibition rate of growth (IG\%) of E. gracilis with stress from different DOM fractions. $\mathbf{f}$ The relationship between $U_{254}$ and IG\% (inset: trendline linear equation and $R^{2}$ value). Asterisk represents $p<0.05$, double asterisk represents $p<0.01$. The values represent mean $\pm S D$, where $n=3$ 
et al. [22]. In this study, we tested the $\mathrm{UV}_{254}$ of 80 -foldconcentrated DOM and found that the fluorescence intensity from high to low was: $\mathrm{HoN}(0.58 \mathrm{AU} / \mathrm{cm}), \mathrm{HoA}$ (0.53 AU/cm), $\mathrm{HiA}(0.50 \mathrm{AU} / \mathrm{cm}), \mathrm{HiB}(0.44 \mathrm{AU} / \mathrm{cm})$, $\mathrm{HoB}(0.30 \mathrm{AU} / \mathrm{cm})$, and $\mathrm{HiN}(0.24 \mathrm{AU} / \mathrm{cm})$ (Fig. 3d). The inhibition of growth, IG\%, of E. gracilis for each of these organic substances were: $28.8 \%, 25.0 \%, 24.6 \%, 19.2 \%$, $12.1 \%$, and $5.0 \%$ (Fig. 3e), respectively. It suggests that all of these DOM fractions can inhibit the growth of $E$. gracilis, especially $\mathrm{HoN}, \mathrm{HoA}$, and $\mathrm{HiA}$. In addition, it is obvious that the $\mathrm{UV}_{254}$ absorption value is linearly related to the IG\% for E. gracilis based on the graph in Fig. $3 \mathrm{f}$ for which $R^{2}=0.9$. The degree of inhibition was positively correlated with the content of luminescent functional groups in the DOM. Based on these results, we confirmed that all fractions of DOM with $\mathrm{C}=\mathrm{O}$ bonds, $\mathrm{C}=\mathrm{C}$ bonds, and aromatic rings have an inhibitory effect on the growth of E. gracilis. In other words, inhibiting the growth of E. gracilis mainly depended on the concentration of different fractions.

According to the above results, DOM mainly includes HA, which was mainly composed of three organic compounds: HiN, HoA, and HoN (Fig. 3b). However, when using U254 signal to characterize these organics, in addition to $\mathrm{HoA}$ and $\mathrm{HoN}$ with relatively high signal intensity, $\mathrm{HiA}, \mathrm{HiB}$, and $\mathrm{HoB}$ also had an inhibitory effect on the growth of E. gracilis, suggesting that inhibitors other than HA may also be present in the recycled culture media. These growth inhibitory factors may be derived from SMBM, FA, and AP (Fig. 3b). These hydrophilic/ hydrophobic fractions also have inhibitory effects on the growth of microalgae, such as FA has been proven to have an inhibitory effect on Scenedesmus species [13], indicating that this fraction, as well as HA with its highly fluorescent signals, may be potential inhibitors. However, both the concentration and the $\mathrm{UV}_{254}$ signal intensity of $\mathrm{HiA}, \mathrm{HiB}$, and $\mathrm{HoB}$ were lower than $\mathrm{HoA}$ and $\mathrm{HoN}$ derived from HA. In addition, although the concentration of HiN derived from HA was relatively higher, it obviously reduced the inhibitory effect on the growth of E. gracilis. Therefore, this study finally confirmed that the main growth inhibition of E. gracilis was HA, and the hydrophobic HoA and HoN organics fractions with higher content and higher $\mathrm{UV}_{254}$ signal intensity played a key inhibitory role. Lu et al. [10] only fractionated HoN-containing fatty acids and showed that they have an inhibitory effect on the growth of S. acuminatus. In addition, Zhang et al. [11] showed that all of the fractions could inhibit the growth of Scenedesmus sp. LX1, especially, $\mathrm{HiB}, \mathrm{HoB}$, and $\mathrm{HiA}$. However, HoN and HoA showed the strongest inhibition of E. gracilis. This suggests that different microalgae may have different tolerances to different classifications of DOM. This scientific problem requires further research.

\section{The influence of $E$. gracilis secretions on its physiology and biochemistry}

The Fv/Fm ratio reflects the ability of microalgae to dissipate, absorb, and transmit light energy during photosynthesis. It is a useful parameter that indicates physiological state and growth rate, and is also an internal probe of the relationship between microalgae and their environment $[13,23,24]$. The Fv/Fm ratio for algae cells was only 0.12 in water containing $\mathrm{HA}$, while that of the control group was 0.64 , which is 5.3 -fold difference (Fig. 4a, $p<0.01)$. From this result, it is obvious that HA significantly reduces the algae cell's photosynthetic efficiency. Similarly, studies on S. acuminatus [13] and Arthrospira platensis [23] also showed comparable Fv/Fm reductions when cultivated in reused water, which means that
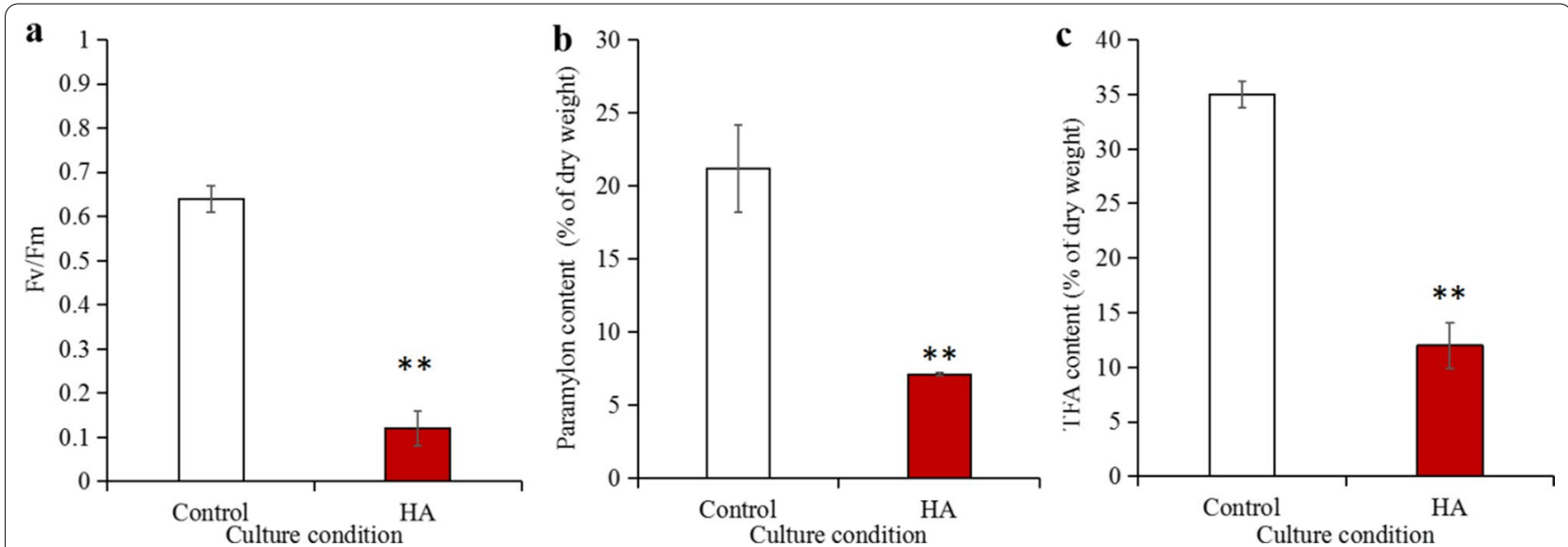

Fig. 4 The effect of self-secreted DOM on (a) Fv/Fm ratio, (b) paramylon content, and (c) TFA content of E. gracilis. TFA total fatty acid, HA humic acid. Double asterisk represents $p<0.01$. The values represent mean \pm SD, where $n=3$ 
HA has a negative effect on the photosynthetic system of these microalgae too. Thus, this impact has a certain universality.

The paramylon content and TFA content of E. gracilis in the experimental group containing DOM were $7.1 \%$ and $12.2 \%$, respectively, while the control group was $21.2 \%$ and $35.2 \%$. Both of these values were significantly lower than the control group, which showed a decrease of $66.5 \%$ (Fig. 4 b, $p<0.01$ ), and $65.3 \%$ (Fig. 4 c, $p<0.01$ ), respectively. These results were confirmed for the TFA of Scenedesmus sp. LX1 [11]. These results indicate that the HA secreted by E. gracilis may interfere with its own photosynthesis, and that this leads to inhibition of the synthesis of organic matter in the algae cells. The mechanism behind this process is worthy of our in-depth study in the future.

\section{Study on the mechanism of E. gracilis growth inhibition by its own secretions}

When UHPLC-QTOF-MS was used to detect metabolites in $E$. gracilis cells and cultivation media, the range of metabolites detected in negative ion mode was greater than that in positive ion mode, so this study only analyzes metabolites that were observed in negative ion mode to describe the mechanism whereby algae cells secrete DOM. With this analysis, we observed 4130 metabolites (Additional file 2). These metabolites were analyzed by PCA and OPLS-DA, and we can see clear separation between intracellular (IEG) and extracellular (EEG) metabolites (Additional file 1: Fig. S2, S3), indicating that there were significant differences in the metabolites in these two groups. When the OPLS-DA permutation test was performed on the data, the categorical variable $Y$ was randomly changed 1000 times (Additional file 1: Fig. S4) and the original model $R^{2} Y$ was equal to 1 , indicating that the established model conforms to the real situation for the sample data. The original model had a $Q^{2}$ value equal to 0.997 , which is very close to 1 . This means that if a new sample were added to the model, it would fall within the existing distribution of data points. In general, the original model is robust and can explain the difference between the two sets of samples well. No overfitting was required to fit our data to it.

This study used VIP $>1$ and a $P$-value $<0.05$ to screen metabolites, and 108 different metabolites were obtained (see Additional file 2). According to the heat map cluster analysis, the relative concentration of 69 and 39 metabolites in the EEG and IEG were up-regulated, respectively (Additional file 1: Fig. S5). After these metabolites were annotated by the KEGG database, important metabolic pathways were screened according to their position and role in the relevant metabolic pathways (Additional file 2). According to the bubble chart, there are nine main metabolic pathways that are relevant: valine, leucine, and isoleucine biosynthesis; linoleic acid metabolism; arginine biosynthesis; the TCA cycle; pyruvate metabolism; purine metabolism; tyrosine metabolism; pyrimidine metabolism; and phenylalanine metabolism (Fig. 5). Among these, the first two are the key metabolic pathways. Some of the metabolites in these metabolic pathways were highly expressed inside the cell, and some were highly expressed outside the cell, and the latter group of metabolites may be secreted from the cell into the cultivation water. Three pathways-linoleic acid metabolism, the TCA cycle, and valine, leucine, and isoleucine biosynthesis-involve $\mathrm{C}=\mathrm{O}$ and $\mathrm{C}=\mathrm{C}$ bonds, while purine and pyrimidine metabolism contribute aromatic rings and $\mathrm{C}=\mathrm{O}$ bonds. These metabolites accumulate in the medium and gradually become $\mathrm{HA}$, which contains various functional groups (Fig. 6).

Palmitic acid was one of the metabolites secreted by $S$. acuminatus to inhibit growth [10]. Similarly, this study found that the palmitic acid in linoleic acid metabolism was higher in concentration in the culture medium. Therefore, it was further proved that palmitic acid was also one of the key factors that inhibit the growth of $E$. gracilis. 2-Isopropylmalate is an intermediate product

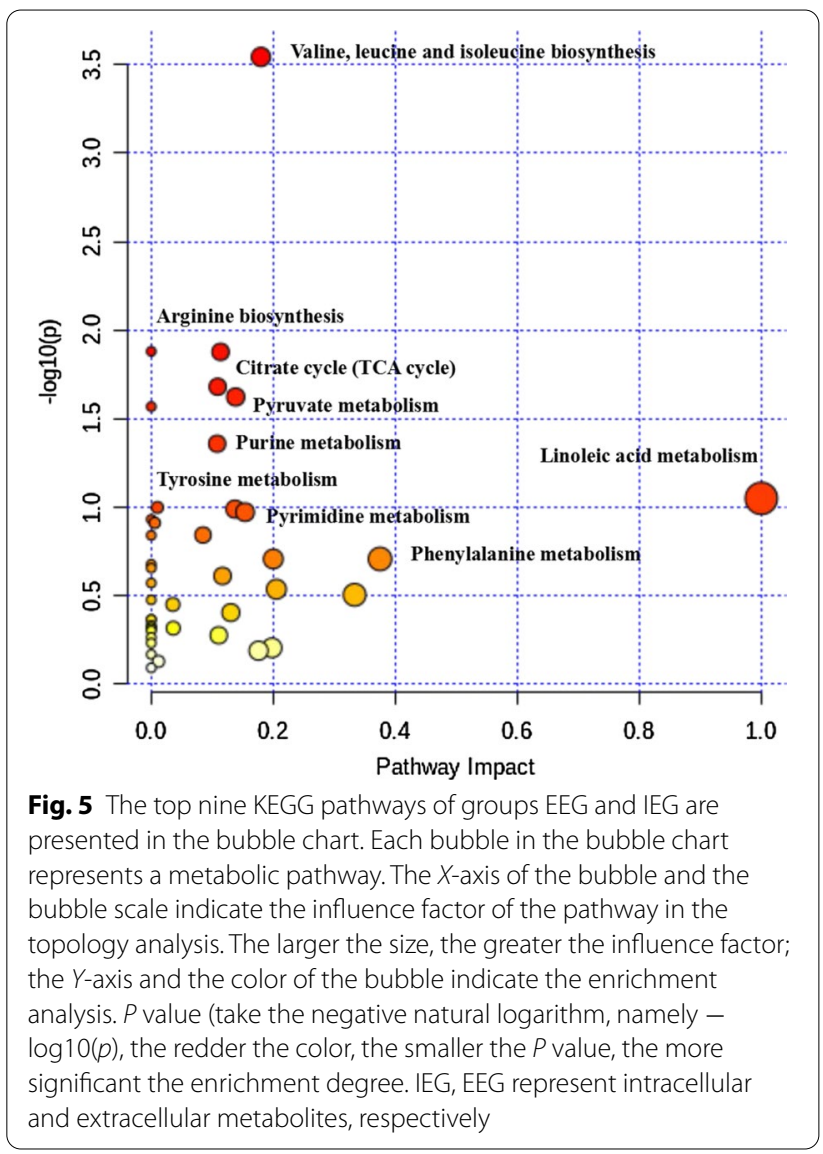




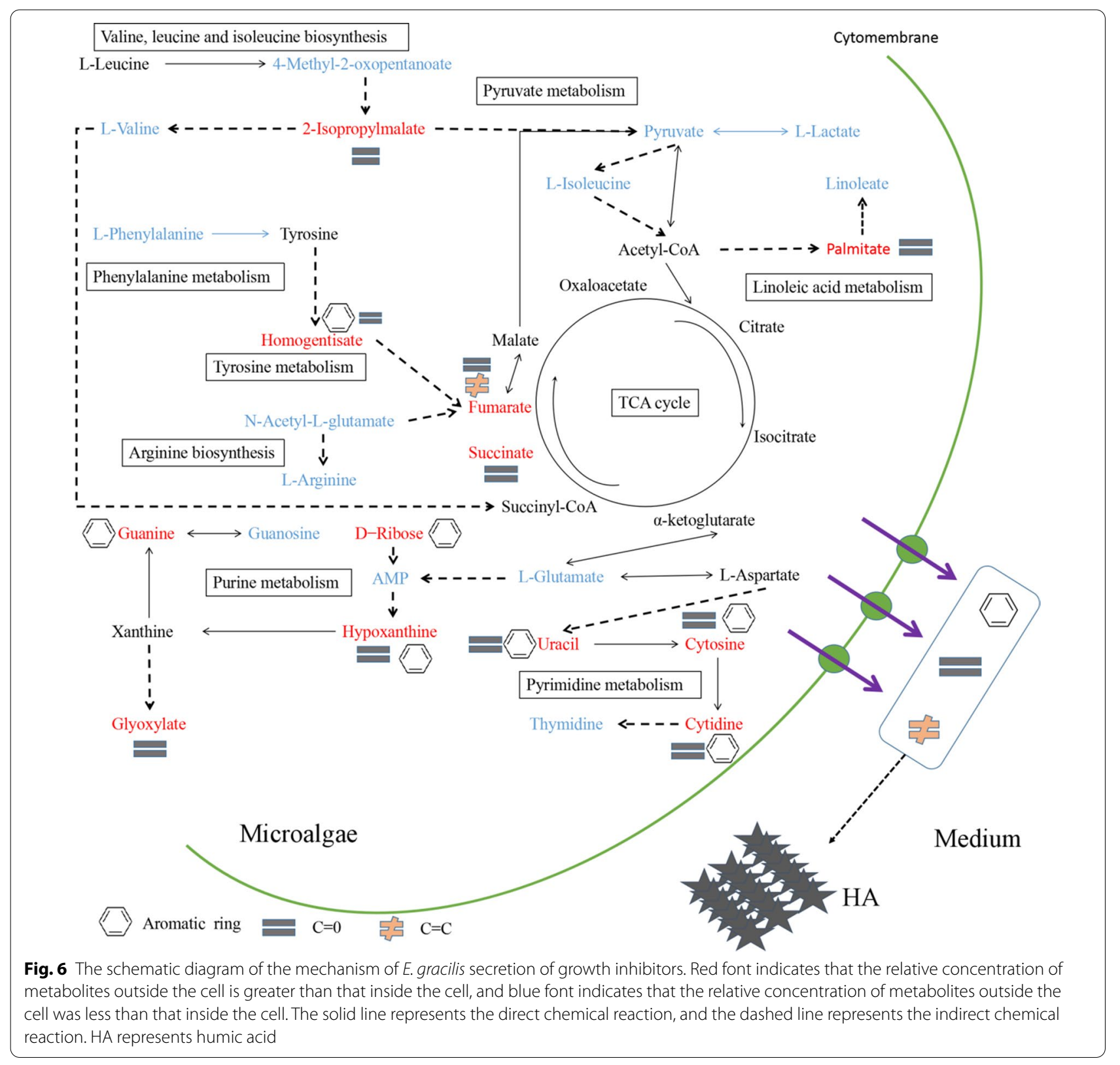

of valine, leucine, and isoleucine biosynthesis. Excessive secretion of this intermediate product from algal cells into the cultivation medium may also inhibit the growth of E. gracilis. However, exactly how this intermediate metabolite inhibits the growth of $E$. gracilis is a question that requires in-depth research in the future.

HA containing multiple functional groups can complex iron ions that are essential for photosynthesis in microalgae. However, Sun et al. [25] found that the underlying mechanism of the inhibitory effect for cyanobacteria was not to reduce the bioavailability of iron, but to inhibit the oxidative damage of cells mediated by peroxidasemediated. More and more evidence shows that HA could directly interact with certain large plants and algae through their different functional groups, thereby interfering with photosynthesis and growth. Due to their low molecular weight $(<50 \mathrm{kDa})$, these substances can easily pass through cell membranes. When these quinone-containing metabolites enter the chloroplast, they interfere with the electron transport processes of photosynthesis $[26,27]$. In fact, the toxic effects of quinones on the growth and photosynthesis of Scenedesmus strains have been confirmed [28]. In addition, we have previously found that there was no significant difference between the experimental group and the control group under heterotrophic conditions containing HA (data not disclosed) 
and the $\mathrm{Fv} / \mathrm{Fm}$ ratio was significantly reduced (Fig. 4a), which means that these inhibitors may primarily attack the photosynthetic system of the $E$. gracilis chloroplast. However, no metabolites related to quinones were found in the different metabolites screened in this study (Additional file 1: Fig. S5), indicating that the photosynthetic machinery of E. gracilis was not affected by quinones. Moreover, it is possible that different functional groups (e.g. $\mathrm{C}=\mathrm{C}$ and $\mathrm{C}=\mathrm{O}$ bonds, aromatic rings) interfere with the electron transport processes of photosynthesis. How these functional groups in the compounds secreted by different metabolic pathways interfere with the photosynthetic system of microalgae requires further in-depth study.

\section{Removal of growth inhibitors}

Markiewicz et al. [29] have confirmed that DOM in sewage is adsorbed effectively by AC. The fluorescence spectrum after $\mathrm{AC}$ treatment showed that the fluorescence signal was very weak (Fig. 7a), indicating that almost all of the HA that can fluoresce had been removed. In addition, the growth curve for the experimental group was almost the same as that of the control group (Fig. 7d). By the time of the last day of culture, the DWs of algal cells were $2.4 \mathrm{~g} / \mathrm{L}$ (experimental group) and $2.4 \mathrm{~g} / \mathrm{L}$ (control group), with no significant difference $(p>0.05)$. This indicates that $\mathrm{AC}$ is effective at completely adsorbing and removing substances that inhibit the growth of E. gracilis. Although the reused water of cultivated N. oceanica [12] and S. acuminatus
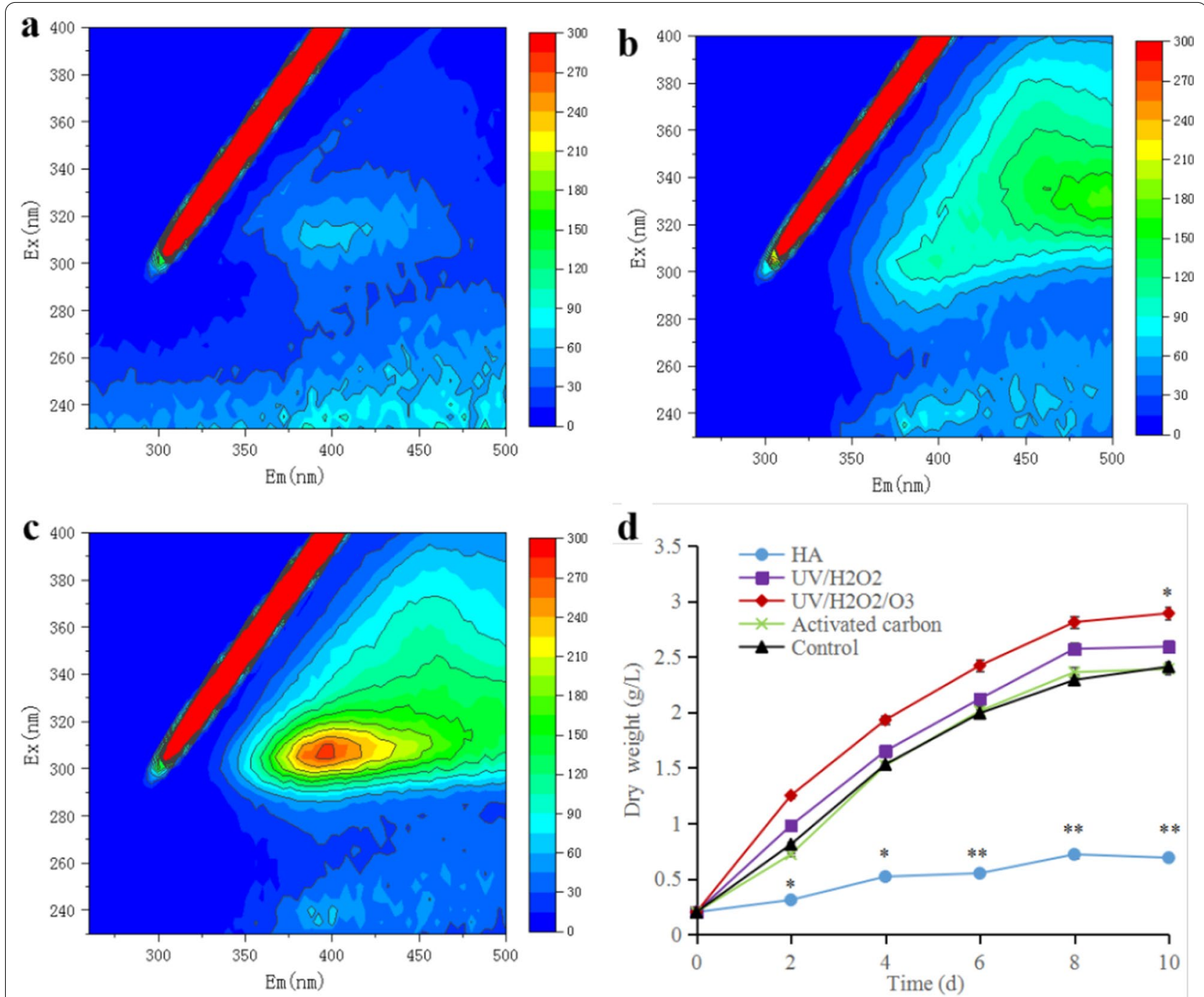

Fig. 7 Removal of growth inhibitors. 3D-FEEM spectra of HA after: (a) activated carbon; (b) $\cup V_{254} / \mathrm{H}_{2} \mathrm{O}_{2} / \mathrm{O}_{3}$-treatment. (c) $U V_{254} / \mathrm{H}_{2} \mathrm{O}_{2}$-treatment; (d) Dry weight (DW) of E. gracilis after different treatments. The regions in these spectra are assigned to various substances found in DOM in Fig. 3b and its caption. HA represents humic acid. Asterisk represents $p<0.05$. The values represent mean \pm SD, where $n=3$ 
[13] showed a relatively significant effect from AC treatment, the biomass obtained was slightly lower than the control group, indicating that some growth inhibitors could not be removed. However, AC effectively adsorbs growth inhibitors secreted by E. gracilis in this study. We would like to develop recyclable AC technology, such as biological $\mathrm{AC}$, to increase the utilization rate so that it can be more convenient for large-scale reuse of water resources to cultivate E. gracilis.

AOPs have been widely used in the field of wastewater treatment. Oxidizers create a large number of free radicals under ultraviolet catalysis, such as hydroxyl radicals. These free radicals have strong oxidizing properties and can oxidize organic acids with unsaturated bonds [15]. According to the 3D-FEEM spectra of the reused water after AOP treatment, the fluorescence signal of the UV/ $\mathrm{H}_{2} \mathrm{O}_{2} / \mathrm{O}_{3}$ group was weaker (Fig. 7b), followed by UV/ $\mathrm{H}_{2} \mathrm{O}_{2}$ (Fig. 7c), indicating that the oxidation efficiency was higher with the participation of $\mathrm{O}_{3}$. In addition, the biomasses of the $\mathrm{UV} / \mathrm{H}_{2} \mathrm{O}_{2} / \mathrm{O}_{3}$ group and the $\mathrm{UV} / \mathrm{H}_{2} \mathrm{O}_{2}$ group on the last day were $2.89 \mathrm{~g} / \mathrm{L}$ and $2.59 \mathrm{~g} / \mathrm{L}$, respectively, with the $\mathrm{UV} / \mathrm{H}_{2} \mathrm{O}_{2} / \mathrm{O}_{3}$ experimental group significantly higher than the control group $(p<0.05)$. These results indicate that the advanced oxidation method not only eliminates the growth inhibitors, but may also oxidize these inhibitors into small organic molecules that could be absorbed by algae cells, thereby increasing their biomass. Our results show that the growth inhibitors were mainly HAs with luminescent functional groups ( $\mathrm{C}=\mathrm{O}$ and $\mathrm{C}=\mathrm{C}$ bonds, aromatic rings). $\mathrm{O}_{3}$ and $\mathrm{UV} /$ $\mathrm{H}_{2} \mathrm{O}_{2}$ have been shown to work well for the treatment of Scenedesmus sp. LX1 [16] and S. acuminatus GT-2 [17] reused water, respectively. This study combines these two methods and shows that both methods together are more effective at removing growth inhibitors than either $\mathrm{O}_{3}$ or $\mathrm{UV} / \mathrm{H}_{2} \mathrm{O}_{2}$ alone. Therefore, we believe that $\mathrm{UV} / \mathrm{H}_{2} \mathrm{O}_{2} / \mathrm{O}_{3}$ is an ideal and efficient method for the removal of inhibitors of E. gracilis.

According to our previous research, the free radicals in the reused water after treatment with AOPs could also inhibit the growth of microalgae. Therefore, we need to optimize the AOP treatment process in the future by optimizing the treatment time, the concentration of the oxidizing agent, and the development of indicators for online detection of the concentration of free radicals in reused water (for example, the vitamin $\mathrm{C}$ reducing agent neutralization method). Use of AOPs is more conducive to the wide application of water reuse for algae cultivation. In addition, through these treatments, again it is clear that HA secreted by E. gracilis is a main growth inhibitor.

Based on the above results, we have proposed a cyclic culture model for E. gracilis (Fig. 8). The conceptual model is optimal when urea replaces $\mathrm{NH}_{4} \mathrm{Cl}$ as a nitrogen source and the reused water is filtered through an UFM and then treated with $\mathrm{UV}_{254} / \mathrm{H}_{2} \mathrm{O}_{2} / \mathrm{O}_{3}$. This model improves the availability of reused water, reduces the cost of cultivation, and increases the biomass of microalga $E$. gracilis.

\section{Conclusion}

Our study demonstrated that cultivation water used three times had a significant inhibitory effect on the growth of E. gracilis. We replaced $\mathrm{NH}_{4} \mathrm{Cl}$ with urea and observed a reduction in the osmotic pressure caused by $\mathrm{Cl}^{-}$accumulation in the reused water, indicating that urea is an ideal nitrogen source. In addition, HA was identified as a main growth inhibitor of E. gracilis, and its content was positively related to the rate of growth inhibition. Moreover, we found that HA interfered with the photosynthetic efficiency of the algae and reduced the efficiency of paramylon and lipid synthesis. We determined that the key metabolic pathways for secreting these HA were valine, leucine, and isoleucine biosynthesis, and linoleic acid metabolism via metabolomics analysis. All HA been efficiently removed or converted into nutrients by $\mathrm{AC}$ or $\mathrm{UV} / \mathrm{H}_{2} \mathrm{O}_{2} / \mathrm{O}_{3}$ treatment, respectively. As a result, the biomass has been recovered to the same levels as the control group (AC treatment) and even enhanced $E$. gracilis growth ( $\mathrm{UV} / \mathrm{H}_{2} \mathrm{O}_{2} / \mathrm{O}_{3}$ treatment). This result provides further confirmation that HA was a main growth inhibitor. An effective model for the cyclic culture of E. gracilis was thus proposed. These studies have important practical and theoretical significance for cyclic cultivation of $E$.

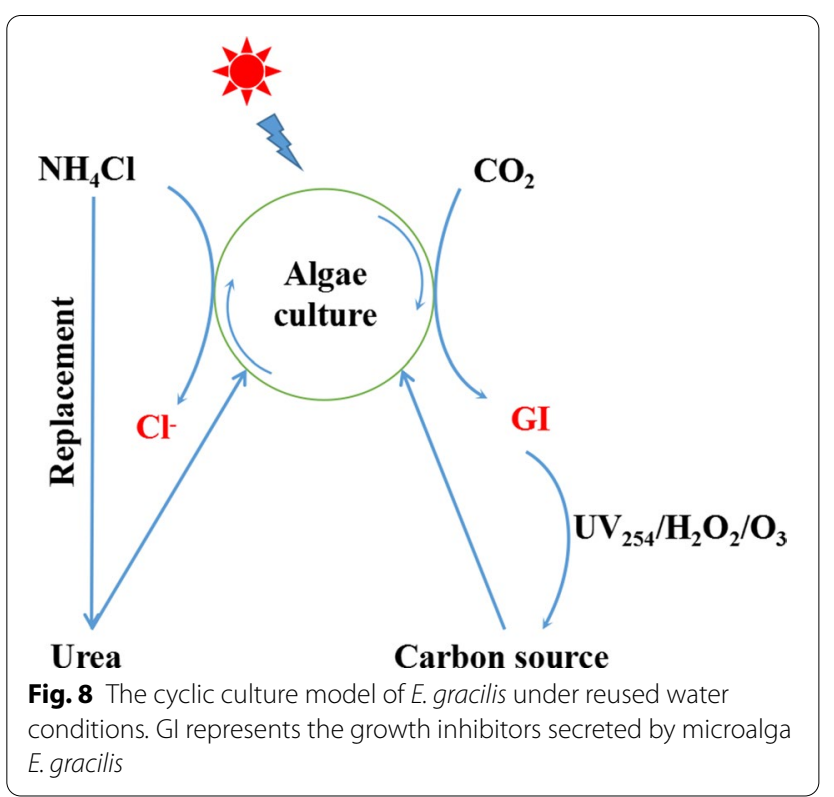


gracilis or even other species of microalgae and for saving precious water resources.

\section{Materials and methods}

\section{Microalgae strain and growth conditions}

Euglena gracilis CCAP 1224/5Z was obtained from the Culture Collection of Algae and Protozoa (CCAP) and maintained in our lab at Shenzhen University [30]. This strain was cultured in a modified photoautotrophic Euglena medium (PEM) according to Cramer and Myers [31]. PEM includes $1.8 \mathrm{~g} / \mathrm{L} \mathrm{NH}_{4} \mathrm{Cl}, 0.6 \mathrm{~g} / \mathrm{L}$ $\mathrm{KH}_{2} \mathrm{PO}_{4}, 1.2 \mathrm{~g} / \mathrm{L} \mathrm{MgSO}_{4} \cdot 7 \mathrm{H}_{2} \mathrm{O}, 0.02 \mathrm{~g} / \mathrm{L} \mathrm{CaCl} \cdot 2 \mathrm{H}_{2} \mathrm{O}$, $0.55 \mu \mathrm{g} / \mathrm{L} \quad \mathrm{Na}_{2}$ EDTA. $2 \mathrm{H}_{2} \mathrm{O}, \quad 2.0 \quad \mu \mathrm{g} / \mathrm{L} \quad \mathrm{Fe}_{2}\left(\mathrm{SO}_{4}\right)_{3}$, $0.05 \mu \mathrm{g} / \mathrm{L} \mathrm{CuSO}_{4} \cdot 5 \mathrm{H}_{2} \mathrm{O}, 0.4 \mu \mathrm{g} / \mathrm{L} \mathrm{ZnSO}_{4} \cdot 7 \mathrm{H}_{2} \mathrm{O}, 1.3 \mu \mathrm{g} / \mathrm{L}$ $\mathrm{Co}\left(\mathrm{NO}_{3}\right)_{2} \cdot 6 \mathrm{H}_{2} \mathrm{O}, 1.8 \mu \mathrm{g} / \mathrm{L} \mathrm{MnCl}_{2} \cdot 4 \mathrm{H}_{2} \mathrm{O}, 0.01 \mu \mathrm{g} / \mathrm{L}$ Vita$\min B_{1}$, and $0.0005 \mu \mathrm{g} / \mathrm{L}$ Vitamin $B_{12}$. The $\mathrm{pH}$ value of the PEM medium was 3.6 adjusted with $3 \mathrm{~mol} / \mathrm{L} \mathrm{NaOH}$ and $1 \mathrm{~mol} / \mathrm{L} \mathrm{HCl}$. The microalgae cells were grown in 2 - L glass column photobioreactors with a $10-\mathrm{cm}$ internal column diameter. The photobioreactors contained $1.5 \mathrm{~L}$ PEM medium that was stirred with $0.2 \mu \mathrm{m}$-filtered mixed gas $\left(2 \% \mathrm{CO}_{2}, \mathrm{v} / \mathrm{v}\right.$, gas flow rate $\left.=6 \mathrm{~L} / \mathrm{min}\right)$ and illuminated with an LED lamp at a light intensity of $150 \mu \mathrm{mol}$ photons $\mathrm{m}^{-2} \mathrm{~s}^{-1}$ (24: $0 \mathrm{~h}$ light-dark cycle). The temperature of the cultivation was maintained at $25.0^{\circ} \mathrm{C}$.

\section{Water reuse}

When the algae cells were cultured on day 10, the cultivation water sample (UFM-R0) was treated with ultrafiltration membrane (UFM) harvesting equipment [32] that was built in our laboratory (Additional file 1: Fig. S1). This equipment cuts off the molecular weight $\geq 50 \mathrm{kDa}$. One equivalent volume of the PEM nutrient medium was added to the UFM-R0 water sample. After sterilization, the solution was inoculated with microalgae to a final $\mathrm{OD}_{750}$ concentration of 0.2 for the microalgae suspension. After the first cultivation, water samples were cycled through this cultivation and UFM cycle three times and these subsequent cycles were named UFM-R1, R2, R3. Samples of the microalgae were taken every other day to monitor the cells' dry weight (DW).

DW was measured according to the method described by $\mathrm{Wu}$ et al. [33]. Briefly, $5 \mathrm{~mL}$ microalgae suspension was filtered through a preheated $\left(105{ }^{\circ} \mathrm{C}, 24 \mathrm{~h}\right)$, preweighed glass microfiber filter (Whatman GF/C, $47 \mathrm{~mm}$, UK). The filters were washed twice, each with $50 \mathrm{~mL}$ of $0.5 \mathrm{~mol} / \mathrm{L} \mathrm{NH}_{4} \mathrm{HCO}_{3}$. The filters were weighed after drying at $105^{\circ} \mathrm{C}$ for $24 \mathrm{~h}$ to reach a constant mass. DW was calculated using Eq. (1):

$$
\text { DW } \frac{\mathrm{g}}{\mathrm{L}}=\frac{\mathrm{w}_{\mathrm{a}}-\mathrm{w}_{\mathrm{b}}}{\mathrm{v}},
$$

where " $w_{\mathrm{a}}$ " and " $w_{\mathrm{b}}$ " are the mass of the filters at the end and start of cultivation, respectively, and " $v$ " is the volume of the microalgae suspension. Finally, the reused water samples and the algae cells were kept at $-80{ }^{\circ} \mathrm{C}$ to prepare for the next study.

\section{The effect of accumulated ions on the growth of E. gracilis}

Algae cells were cultured in PEM with nutrient concentrations of 10, 9, 8, 7, 6, 5, 4, 3, 2, 1, 0.5, and 0.2-fold, with $1.8 \mathrm{~g} / \mathrm{L} \mathrm{NH}_{4} \mathrm{Cl}$ or $1.0 \mathrm{~g} / \mathrm{L}$ urea (both of them contained equal nitrogen content) as the nitrogen source. When the algae cells were cultured on day 10, the morphology of the algae cells was observed with an inverted microscope (Leica DMI8, Leica Microsystems, Germany). In addition, the DW, $\mathrm{Cl}^{-}$concentration, salinity, osmolality, and the pigment changes of the algae cells in the culture medium were measured. $\mathrm{Cl}^{-}$concentration was measured using a capillary ion chromatograph (ICS 5000+, Dionex, Sunnyvale, CA, USA) [10]. The salinity of the culture medium was measured using an Orion STAR A329 multiparameter meter (G10919, Thermo Fisher Scientific, USA). Osmolality was determined by measuring the freezing point of the solution with an automatic osmometer (Osmomat 030, Gonotec, Germany) according to Hadj-Romdhane et al. [9]. The osmolality was calculated using the freezing point depression, $\Delta T\left({ }^{\circ} \mathrm{C}\right)$, according to the following Eq. (2):

$$
\text { Osmolalitymosm }=\frac{\Delta \mathrm{T}\left({ }^{\circ} \mathrm{C}\right)}{1.858},
$$

where 1.858 is the cryoscopic constant, which is equal to the freezing point depression of a solution with an osmolality of 1 osmol.

\section{The identification of $E$. gracilis growth inhibitors and quantitative analysis of their effect on growth}

The differences in dissolved organic matter (DOM) content between the control (UFM-R0) and the reused water samples (UFM-R1, R2, R3) were measured using a total organic carbon analyzer (Multi N/C 2100, Analytik Jena, Germany).

DOM was determined with three-dimensional fluorescence excitation-emission matrix (3D-FEEM) spectrophotometry. Briefly, 3D-FEEM spectra were obtained using a fluorescence spectrophotometer (F-4500, Hitachi, Japan). The excitation (Ex) and emission (Em) slits were set to a bandpass of $5 \mathrm{~nm}$. Ex wavelengths were scanned from 200 to $450 \mathrm{~nm}$, and Em wavelengths were scanned from 220 to $550 \mathrm{~nm}$. All of the 3D-FEEM spectral data were analyzed with Origin Pro 2018 software (https:// www.originlab.com/origin). 
The method of pretreated water sample was based on Leenheer [34]. The fractional method of DOM from water sample was optimized by Imai et al. [35] and Zhang et al. [36]. Briefly, the water sample was repeatedly passed through Amberlite XAD- 8 resin at a flow rate of $5 \mathrm{~mL} /$ min 3 times, and then 2 bed volumes (BV) of $0.1 \mathrm{~mol} / \mathrm{L}$ $\mathrm{HCl}$ were added to reverse the elution 3 times to obtain the hydrophobic bases $(\mathrm{HoB})$. The $\mathrm{pH}$ of the water sample was adjusted to 2.0 using $0.1 \mathrm{~mol} / \mathrm{L} \mathrm{HCl}$ and $0.1 \mathrm{~mol} / \mathrm{L}$ $\mathrm{NaOH}$, then the water sample was passed through 3 columns with XAD-8 resin, D001(a microporous strong acidic ion exchange resin), and D201 (a macroporous strong base ion exchange resin) orderly. This sequence was repeated 3 times. After the water sample was passed through the resin, the remaining water sample contained only the hydrophilic neutrals (HiN). The XAD-8, D001, and D201 resins were back-eluted with $0.1 \mathrm{~mol} / \mathrm{L} \mathrm{NaOH}$ to obtain hydrophobic acids (HoA), hydrophilic bases (HiB), and hydrophilic acids (HiA), respectively. After the XAD-8 resin was air dried, ethanol was added and soxhlet extraction was run for $12 \mathrm{~h}$ to obtain the hydrophobic neutrals $(\mathrm{HoN})$. All solvents were removed using rotary-evaporation (RV3, IKA, Germany). The volume of all DOM fractions was adjusted to $50 \mathrm{~mL}$ and transferred to centrifuge tubes. After diluting each group of DOM to a certain concentration, the percentage of the DOM in each fraction was measured by using a total N/C analyser (Multi N/C 2100, Analytik Jena, Germany). An absorbance value of $254 \mathrm{~nm}\left(\mathrm{UV}_{254}\right)$ of DOM in each fraction was measured by using a UV-Vis spectroscopic measurements (UV2350, UNICO, China).

The fractionated organic acids were added to fresh medium according to the percentage of DOM. After culturing the algae cells with this medium for 10 days, the DWs of the control and the experimental groups were measured. The inhibition rate of growth (IG\%) was calculated using the following Eq. (3):

$$
\mathrm{IG} \%=\frac{\mathrm{c}-\mathrm{i}}{\mathrm{c}} \times 100 \%,
$$

where " $c$ " is the DW of the control group and " $i$ " is the DW of algae cells under the different fractionated DOM stressed conditions. Finally, the correlation analysis of $\mathrm{UV}_{254}$ and IG\% were performed by using Origin Pro 2018 software.

\section{The effect of growth inhibitors on the Fv/Fm ratio, paramylon and total fatty acid content}

After the fractionated DOM was diluted according to the DOM content in the UFM-R3 reused water, as described above, fresh medium was added, and then the algae cells were cultivated under the afore mentioned culture conditions. The Fv/Fm ratio, the paramylon and the total fatty acid (TFA) content of the algae cells were measured after the 10th day of cultivation.

The $\mathrm{Fv} / \mathrm{Fm}$ ratio was determined by dividing the variable fluorescence $(\mathrm{Fv})$ by the maximum fluorescence $(\mathrm{Fm})$, according to the method of Sha et al. [13]. The algae cells were placed in a quartz cube and maintained in the dark for $3 \mathrm{~min}$ prior to measurement of $\mathrm{Fv} / \mathrm{Fm}$. The $\mathrm{Fv} / \mathrm{Fm}$ ratio for the algae cells was then measured at room temperature using a PHYTO-ED fluorimeter (Walz, Effeltrich, Germany).

Paramylon content was quantified using the method of Takenaka et al. [37] and Wu et al. [30] with the following modification: $2 \mathrm{~mL}, 30 \mathrm{mmol} / \mathrm{L}$ of EDTA chelating agent was added to $15 \mathrm{~mL}$ the algae cells suspensions. After each cell suspension was centrifuged and freezedried, $10 \mathrm{mg}$ freeze-dried algae powder and $5 \mathrm{~mL}$ of acetone were transferred to a $15 \mathrm{~mL}$ of centrifuge tube and shaken for $30 \mathrm{~s}$, then placed in a shaker for $2 \mathrm{~h}$. After the tube was centrifuged at $2000 \times g$ for $5 \mathrm{~min}$, the supernatant was removed. $1.5 \mathrm{~mL}$ of $1 \%$ sodium dodecyl sulfate (SDS) solution was added to the tube, then the contents were transferred into a $1.5-\mathrm{mL}$ centrifuge tube and heated in a water bath at $85^{\circ} \mathrm{C}$ for $2 \mathrm{~h}$. Again, the supernatant was removed after the centrifuge tube was centrifuged at $2000 \times g$ for $5 \mathrm{~min}$. The precipitate was washed and centrifuged in $1 \mathrm{~mL}$ deionized water, then oven-dried at $70{ }^{\circ} \mathrm{C}$ to a constant mass. The resulting precipitate was paramylon. The paramylon content was calculated as shown in Eq. (4):

$$
\text { Paramyloncontent } \%=\frac{\mathrm{P}}{\mathrm{DW}} \times 100 \%
$$

where "P" and "DW" are the DWs of the paramylon and the algae powder, respectively.

TFA content was determined using the method of Wu et al. [38]. Briefly, about $10 \mathrm{mg}$ lyophilized cell pellets were disrupted by grinding three times under liquid nitrogen in the presence of methanol, chloroform, and formic acid (20:10:1, v:v:v) to extract the lipids from the algal biomass. The quantity of TFA content in extracts was measured by using an Agilent 7890B gas chromatograph coupled with a 5977A mass spectrometer (GC-MS).

\section{The metabolic pathways of growth inhibitors secreted by $E$. gracilis were determined using metabolomics analysis}

$E$. gracilis cells and cultivation water from UFM-R0 were collected and a metabolomics analysis was performed. The metabolites in the sample were extracted and analyzed according to the method mentioned by Wu et al. [38]. All of the metabolites were detected using ultra-high performance liquid chromatography coupled with quadrupole time-of-flight mass spectrometry 
(UHPLC-QTOF-MS). In this study, some metabolite peaks were detected after relative standard deviation noise reduction. Next, the missing values were increased by half of the minimum value. An internal standard normalization method was also employed in this data analysis. The final dataset containing the peak number, sample name, and normalized peak area was imported to a SIMCA16.0.2 software package (Sartorius Stedim Data Analytics AB, Umea, Sweden) for multivariate analysis. Data was scaled and logarithmically transformed to minimize the impact of both noise and high variance of the variables. After these transformations, principal component analysis (PCA), an analysis that reduces the dimensionality of the data, was carried out to visualize the distribution and the grouping of the samples. A 95\% confidence interval in the PCA score plot was used as the threshold to identify potential outliers in the dataset. In order to visualize group separation and find significantly changed metabolites, orthogonal-projections-to-latentstructures discriminate analysis (OPLS-DA) was applied. Then a sevenfold cross-validation was performed to calculate the values of $R^{2}$ and $Q^{2} \cdot R^{2}$ indicates how well the data variance fits the model, and $Q^{2}$ indicates how well a variable can be predicted. To check the robustness and predictive ability of the OPLS-DA model, 1000 permutations were further conducted. Afterward, the $R^{2}$ and $Q^{2}$ intercept values were obtained. The intercept value of $Q^{2}$ represents the robustness and reliability of the model and the risk of overfitting (for the latter, smaller values are better). Furthermore, the value of variable importance in the projection (VIP) of the first principal component in OPLS-DA analysis was obtained. It summarizes the contribution of each variable to the model. The metabolites with VIP $>1$ and $p<0.05$ (Student's $t$-test) were considered to be significantly changed. In addition, commercial databases including KEGG database (http://www. genome.jp/kegg/) and MetaboAnalyst (http://www. metaboanalyst.ca/) were used for metabolic pathway enrichment analysis. From these analyses, bubble diagrams and metabolic pathways were made.

\section{Removal of growth inhibitors}

The methods of advanced oxidation that we used (UV/ $\mathrm{H}_{2} \mathrm{O}_{2} / \mathrm{O}_{3}$ and $\mathrm{UV} / \mathrm{H}_{2} \mathrm{O}_{2}$ ) were similar to the result of $\mathrm{Hu}$ et al. [16] and Wang et al. [17]. Briefly, an ozone generator, which produced $\mathrm{O}_{3}$ with a flow rate of $3000 \mathrm{mg} / \mathrm{h}$, was fed into a solution containing $189.2 \mathrm{mg} / \mathrm{L} \mathrm{DOM}$ (found in reused water UFM-R3) and $1 \% \mathrm{H}_{2} \mathrm{O}_{2}$ for $2 \mathrm{~h}$ under $\mathrm{UV}_{254}$ ultraviolet lamp irradiation. This is the $\mathrm{UV} / \mathrm{H}_{2} \mathrm{O}_{2} / \mathrm{O}_{3}$ experimental group. In the other set of experiments, $\mathrm{O}_{3}$ was not used, but all other conditions were the same. This is the $\mathrm{UV} / \mathrm{H}_{2} \mathrm{O}_{2}$ experimental group. After all the treated water samples were freeze-dried, fresh medium was added to each. The method of AC filtration was similar to that of Sha et al. [13]. Briefly, the water sample containing the same UFM-R3 DOM concentration was filtered repeatedly through a chromatography column containing saturated AC (K04, Hainan Xingguang Active Carbon Co., LTD. China) for $2 \mathrm{~h}$. Then 0.45 -micron membrane filters were used to recover the water sample. DOM was characterized by using 3D-FEEM spectroscopy. Finally, the microalgae cells were cultured under the experimental conditions, and the DW of the algae cells was measured every other day.

\section{Statistical analysis}

All DWs, $\mathrm{Cl}^{-}$concentration, salinity, osmolality, DOM, $\mathrm{UV}_{254}, \mathrm{IG} \%$, Fv/Fm ratio, paramylon content, and TFA content tests were performed in triplicate and the average and standard deviations was reported. All data were statistically analyzed by Student's $t$-test analysis to investigate the difference between the control and experimental groups. $p$-values of less than $0.01(p<0.01)$ were considered significantly different, $p<0.05$ values were considered statistically different, and $p>0.05$ values were considered not statistically different (NS) compared to the control groups.

\section{Abbreviations \\ UFM: Ultrafiltration membrane; AC: Activated carbon; AOPs: Advanced oxidation processes; HA: Humic acid; DOM: Dissolved organic matter; AP: Aromatic proteins; FA: Fulvic-acid-like substances; SMBM: Soluble microbial by product-like material; IEG: Intracellular; EEG: Extracellular; CCAP: Culture Collection of Algae and Protozoa; PEM: Photoautotrophic Euglena medium; 3D-FEEM: Three-dimensional fluorescence excitation-emission matrix; Em: Excitation (Ex) and emission; DW: Cells' dry weight; HoB: Hydrophobic bases: BV: Bed volumes; HoB: Hydrophobic bases; HiN: Hydrophilic neutrals; HoA: Hydrophobic acids; HiB: Hydrophilic bases; HiA: Hydrophilic acids; HoN: Hydro- phobic neutrals; IG\%: The inhibition rate of growth; TFA: The total fatty acid; Fm: The maximum fluorescence; GC-MS: Gas chromatograph coupled with a 5977A mass spectrometer; UHPLC-QTOF-MS: Quadrupole time-of-flight mass spectrometry; PCA: Principal component analysis; OPLS-DA: Orthogonal- projections-to-latent-structures discriminate analysis; VIP: The value of variable importance in the projection; NS: Statistically different.}

\section{Supplementary Information}

The online version contains supplementary material available at https://doi. org/10.1186/s13068-021-01980-4.

Additional file 1: Fig. S1. Microalgae harvesting equipment. a, microalgae suspension; $b$, ultrafiltration membrane; $c$, pre-concentrated microalgae; d, reused water. Fig. S2. PCA analysis for groups EEG and IEG. IEG, EEG represent intracellular and extracellular metabolites, respectively. Fig. S3. OPLS-DA analysis for group EEG and IEG. IEG, EEG represent intracellular and extracellular metabolites, respectively. Fig. S4. Permutation test for group EEG and IEG. IEG, EEG represent intracellular and extracelIular metabolites, respectively. Fig. S5. Heatmap of hierarchical clustering analysis for group EEG and IEG. IEG, EEG represent intracellular and extracellular metabolites, respectively; * represents the metabolite can be annotated in KEGG database.

Additional file 2. The data of statistical analysis results, different metabolites, and pathway analysis from the group EEG and IEG. 


\section{Acknowledgements}

We acknowledge TopEdit LLC (www.topeditsci.com) for the linguistic editing and proofreading during the preparation of this manuscript. We thank Instrument Analysis Center of Shenzhen University for the assistance with an inverted microscope (Leica DMI8, Leica Microsystems, Germany) analysis.

\section{Authors' contributions}

MW conceived and designed the experiments; MW and JL performed the experiments; MW analyzed the data; MW, MD, GW, FL, JW, AL, ZH and HZ wrote and revised the manuscript. All authors read and approved the final manuscript.

\section{Funding}

This work was supported by the National Natural Science Foundation of China (Grant numbers 31670116, 41876188), Guangxi Innovation Drive Development Special Fund (Gui Ke AA18242047) and Grant Plan for Demonstration Project for Marine Economic Development in Shenzhen to Dr. Zhangli Hu; the Natural Science Foundation of Guangdong Province, China (2014A030313562), National Key R\&D Program of China (2018YFA0902500), the Guangdong Innovation Research Team Fund (Grant number, 2014ZT05S078), and the Shenzhen Grant Plan for Science and Technology (Grant numbers, JCYJ20160308095910917, JCYJ20170818100339597).

\section{Availability of data and materials}

All data generated or analyzed in the present study are included in this article and in additional information.

\section{Declarations}

\section{Ethics approval and consent to participate}

Not applicable.

\section{Consent for publication}

All authors agree with submission to Biotechnology for Biofuel.

\section{Competing interests}

There are no competing interests associated with this manuscript.

\section{Author details}

'Shenzhen Key Laboratory of Marine Bioresource and Eco-Environmental Science, Shenzhen Engineering Laboratory for Marine Algal Biotechnology, Guangdong Provincial Key Laboratory for Plant Epigenetics, College of Life Sciences and Oceanography, Shenzhen University, Shenzhen 518060, China. ${ }^{2}$ Key Laboratory of Optoelectronic Devices and Systems of Ministry of Education and Guangdong Province, College of Optoelectronic Engineering, Shenzhen University, Shenzhen 518060, China. ${ }^{3}$ College of Food Engineering and Biotechnology, Hanshan Normal University, Chaozhou 521041, China.

Received: 29 December 2020 Accepted: 26 May 2021

Published online: 05 June 2021

\section{References}

1. Deviram G, Mathimani T, Anto S, Ahamed TS, Ananth DA, Pugazhendhi A. Applications of microalgal and cyanobacterial biomass on a way to safe, cleaner and a sustainable environment. J Clean Prod. 2020;253:119770.

2. Martinez AKA, Lauritano C, Romano G, lanora A. Marine microalgae with anti-cancer properties. Mar Drugs. 2018;16:165.

3. Lu Z, Loftus S, Sha J, Wang W, Park MS, Zhang X, Johnson ZI, Hu Q. Water reuse for sustainable microalgae cultivation: current knowledge and future directions. Resources Conser Recy. 2020;161:104975.

4. Shahid A, Malik S, Zhu H, Xu J, Nawaz MZ, Nawaz S, Alam MA, Mehmood MA. Cultivating microalgae in wastewater for biomass production, pollutant removal, and atmospheric carbon mitigation. Sci Total Environ. 2020;704:135303

5. Junior EN, Kumar M, Pankratz S, Oyedun AO, Kumar A. Development of life cycle water footprints for the production of fuels and chemicals from algae biomass. Water Res. 2018;140:311-22.
6. Loftus SE, Johnson ZI. Cross-study analysis of factors affecting algae cultivation in recycled medium for biofuel production. Algal Res. 2017;24:154-66.

7. Kottuparambil S, Thankamony RL, Agusti S. Euglena as a potential natural source of value-added metabolites. Algal Res. 2019;37:154-9.

8. Discart V, Bilad MR, Marbelia L, Vankelecom IF. Impact of changes in broth composition on Chlorella vulgaris cultivation in a membrane photobioreactor (MPBR) with permeate recycle. Bioresour Technol. 2014;152:321-8.

9. Hadj-Romdhane F, Jaouen P, Pruvost J, Grizeau D, Van Vooren G, Bourseau P. Development and validation of a minimal growth medium for recycling Chlorella vulgaris culture. Bioresour Technol. 2012;123:366-74.

10. Lu Z, Sha J, Wang W, Li Y, Wang G, Chen Y, Hu Q, Zhang X. Identification of auto-inhibitors in the reused culture media of the Chlorophyta Scenedesmus acuminatus. Algal Res. 2019;44:101665.

11. Zhang TY, Yu Y, Wu YH, Hu HY. Inhibitory effects of soluble algae products (SAP) released by Scenedesmus sp. LX1 on its growth and lipid production. Bioresour Technol. 2013;146:643-8.

12. Zhang $X$, Lu Z, Wang Y, Wensel P, Sommerfeld M, Hu Q. Recycling Nannochloropsis oceanica culture media and growth inhibitors characterization. Algal Res. 2016;20:282-90.

13. Sha J, Lu Z, Ye J, Wang G, Hu Q, Chen Y, Zhang X. The inhibition effect of recycled Scenedesmus acuminatus culture media: influence of growth phase, inhibitor identification and removal. Algal Res. 2019;42:101612.

14. Miklos DB, Remy C, Jekel M, Linden KG, Drewes JE, Hubner U. Evaluation of advanced oxidation processes for water and wastewater treatment. Water Res. 2018;139:118-31.

15. Zhang $R$, Sun $P$, Boyer $T H$, Zhao L, Huang $C H$. Degradation of pharmaceuticals and metabolite in synthetic human urine by UV, UV/ $\mathrm{H}_{2} \mathrm{O}_{2}$, and UV/ PDS. Environ Sci Technol. 2015;49:3056-66.

16. Hu HY, Zhang TY, Shi XJ, Wu YH, Zhuang LL. Using ozonation to eliminate the inhibition of soluble algal products (SAP) of Scenedesmus sp. LX1 on its growth in microalgal cultivation for biomass/bioenergy production. Water Sup. 2015;15:1034-9.

17. Wang W, Sha J, Lu Z, Shao S, Sun P, Hu Q, Zhang X. Implementation of UVbased advanced oxidation processes in algal medium recycling. Sci Total Environ. 2018;634:243-50.

18. Yu Z, Pei H, Hou Q, Nie C, Zhang L, Yang Z, Wang X. The effects of algal extracellular substances on algal growth, metabolism and long-term medium recycle, and inhibition alleviation through ultrasonication. Bioresour Technol. 2018:267:192-200.

19. Tao R, Lakaniemi AM, Rintala JA. Cultivation of Scenedesmus acuminatus in different liquid digestates from anaerobic digestion of pulp and paper industry biosludge. Bioresource Technol. 2017;245:706-13.

20. Sheng GP, Yu HQ. Characterization of extracellular polymeric substances of aerobic and anaerobic sludge using three-dimensional excitation and emission matrix fluorescence spectroscopy. Water Res. 2006;40:1233-9.

21. Chen W, Westerhoff P, Leenheer JA, Booksh K. Fluorescence excitationemission matrix regional integration to quantify spectra for dissolved organic matter. Environ Sci Technol. 2003;37:5701-10.

22. Altmann J, Massa L, Sperlich A, Gnirss R, Jekel M. UV ${ }_{254}$ absorbance as real-time monitoring and control parameter for micropollutant removal in advanced wastewater treatment with powdered activated carbon. Water Res. 2016:94:240-5.

23. Depraetere O, Pierre G, Noppe W, Vandamme D, Foubert I, Michaud P, Muylaert K. Influence of culture medium recycling on the performance of Arthrospira platensis cultures. Algal Res. 2015;10:48-54.

24. Oukarroum A. Change in photosystem II photochemistry during algal growth phases of Chlorella vulgaris and Scenedesmus obliquus. Curr Microbiol. 2016;72:692-9.

25. Sun BK, Tanji Y, Unno H. Extinction of cells of cyanobacterium Anabaena circinalis in the presence of humic acid under illumination. Appl Microbiol Biot. 2006;72:823.

26. Steinberg CEW, Kamara S, Prokhotskaya VY, Manusadzianas L, Karasyova TA, Timofeyev MA, Jie Z, Paul A, Meinelt T, Farjalla VF, Matsuo AYO, Kent Burnison B, Menzel R. Dissolved humic substances-ecological driving forces from the individual to the ecosystem level? Freshwater Biol. 2006;51:1189-210.

27. Steinberg CEW, Timoveyev M, Menzel R. Dissolved humic substances: interactions with organisms. Encycloped Inland Waters. 2009;747-753. 
28. Tukaj Z, Aksmann A. Toxic effects of anthraquinone and phenanthrenequinone upon Scenedesmus strains (green algae) at low and elevated concentration of $\mathrm{CO}_{2}$. Chemosphere. 2007;66:480-7.

29. Markiewicz A, Stromvall AM, Bjorklund K. Alternative sorption filter materials effectively remove non-particulate organic pollutants from stormwater. Sci Total Environ. 2020;730:139059.

30. Wu M, Li J, Qin H, Lei A, Zhu H, Hu Z, Wang J. Pre-concentration of microalga Euglena gracilis by alkalescent $\mathrm{pH}$ treatment and flocculation mechanism of $\mathrm{Ca}_{3}\left(\mathrm{PO}_{2}\right)_{2}, \mathrm{Mg}_{3}\left(\mathrm{PO}_{4}\right)_{2}$, and derivatives. Biotechnol Biofuels. 2020;13:98.

31. Cramer M, Myers J. Growth and photosynthetic characteristics of Euglena Gracilis. Arch Mikrobiol. 1952;17:384-402.

32. Wang L, Pan B, Gao Y, Li C, Ye J, Yang L, Chen Y, Hu Q, Zhang X. Efficient membrane microalgal harvesting: pilot-scale performance and technoeconomic analysis. J Clean Prod. 2019;218:83-95.

33. Wu M, Zhu R, Lu J, Lei A, Zhu H, Hu Z, Wang J. Effects of different abiotic stresses on carotenoid and fatty acid metabolism in the green microalga Dunaliella salina Y6. Ann Microbiol. 2020;70.

34. Leenheer JA. Comprehensive approach to preparative isolation and fractionation of dissolved organic carbon from natural waters and wastewaters. Environ Sci Technol. 1981;15:578-87.
35. Imai A, Fukushima T, Matsushige K, Kim YH, Choi K. Characterization of dissolved organic matter in effluents from wastewater treatment plants. Water Res. 2002;36:859-70.

36. Zhang H, Qu J, Liu H, Zhao X. Characterization of isolated fractions of dissolved organic matter from sewage treatment plant and the related disinfection by-products formation potential. J Hazard Mater (2-3). 2009; 164:1433-8

37. Takenaka S, Kondo T, Nazeri S, Tamura Y, Tokunaga M, Tsuyama S, Miyatake $\mathrm{K}$, Nakano Y. Accumulation of trehalose as a compatible solute under osmotic stress in Euglena gracilis Z. J Eukaryot Microbiol. 1997;44:609-13.

38. Wu M, Zhang H, Sun W, Li Y, Hu Q, Zhou H, Han D. Metabolic plasticity of the starchless mutant of Chlorella sorokiniana and mechanisms underlying its enhanced lipid production revealed by comparative metabolomics analysis. Algal Res. 2019;42:101587.

\section{Publisher's Note}

Springer Nature remains neutral with regard to jurisdictional claims in published maps and institutional affiliations.
Ready to submit your research? Choose BMC and benefit from:

- fast, convenient online submission

- thorough peer review by experienced researchers in your field

- rapid publication on acceptance

- support for research data, including large and complex data types

- gold Open Access which fosters wider collaboration and increased citations

- maximum visibility for your research: over $100 \mathrm{M}$ website views per year

At BMC, research is always in progress.

Learn more biomedcentral.com/submissions 